\title{
El diseño de la instrucción para la comprensión lectora en Educación Primaria: un estudio de caso
}

Arreola Saldívar, Elizabeth; Coronado Manqueros, Juan Manuel

El diseño de la instrucción para la comprensión lectora en Educación Primaria: un estudio de caso

Revista Educación, vol. 45, núm. 1, 2021

Universidad de Costa Rica, Costa Rica

Disponible en: http://www.redalyc.org/articulo.oa?id=44064134023

DOI: https://doi.org/10.15517/revedu.v45i1.41326

Esta obra está bajo una Licencia Creative Commons Atribución-NoComercial-SinDerivar 3.0 Internacional. 


\title{
El diseño de la instrucción para la comprensión lectora en Educación Primaria: un estudio de caso
}

\author{
Designing Reading Comprehension Instruction for Primary Education: A Case Study
}

Elizabeth Arreola Saldivar

Universidad Pedagógica de Durango, México

mi_b_bk@hotmail.com

iD http://orcid.org/0000-0003-4708-2552

Juan Manuel Coronado Manqueros

Universidad Pedagógica de Durango, México

jmcoronadom@gmail.com

\author{
DOI: https://doi.org/10.15517/revedu.v45i1.41326 \\ Redalyc: http://www.redalyc.org/articulo.oa?id=44064134023
}

Recepción: 23 Abril 2020

Aprobación: 22 Septiembre 2020

\section{RESUMEN:}

La comprensión lectora es un proceso cognitivo que involucra un gran número de tareas mentales simultáneas; este proceso demanda una seria atención al detalle del cómo lograrla en el estudiantado, es aquí donde el Diseño Instruccional (DI) para su fomento cobra relevancia. El presente estudio se plantea el objetivo de recuperar y analizar la manera en la cual el profesorado se prepara previamente para centrar su atención en la comprensión lectora de sus estudiantes, es decir, de qué manera diseñan su instrucción pensando en las cuestiones como los objetivos, valores, métodos, herramientas y actividades que desarrolla la comprensión lectora. Para ello se elabora una investigación cualitativa con el estudio de caso (Stake, 1998) como metodología y se utiliza la entrevista semiestructurada como técnica de recolección de datos, la cual se aplicó a dos docentes que se desempeñan en educación primaria, superior y posgrado en escuelas públicas y privadas de la ciudad de Durango, Durango, ambos con grado de doctor en Ciencias para el Aprendizaje y con una amplia experiencia en el campo de la cognición; mientras que el análisis de la información se efectuó siguiendo el procedimiento denominado inducción analítica. Entre los hallazgos se puede advertir que el profesorado de educación primaria diseña y lleva a la práctica del aula de clases diversas actividades, herramientas y estrategias relacionadas con el objetivo implícito de buscar el desarrollo de la comprensión lectora en diversos momentos, lo que permite comprender que sea el DI la herramienta primordial para influir en el perfeccionamiento de la compresión lectora. La investigación demuestra que un/a docente informado/a y bien preparado/a para la tarea del diseño instruccional hace la diferencia en el logro de aprendizajes y de la comprensión lectora.

Palabras Clave: Comprensión, Lectura, Diseño, Instrucción, Cognición.

\section{Abstract:}

Reading comprehension is a cognitive process involving a large number of simultaneous mental tasks. The ability to teach students this skill requires paying close attention to detail and use of Instructional Design (ID). This study endeavors to recover and analyze how teachers prepare beforehand to teach Reading Comprehension. This includes how they design instructional thought regarding objectives, values, methods, tools and activities to develop this skill. A qualitative investigation was conducted using the methodology of a case study (Stake, 1998). Data collection was based on a semi-structured interview of two teachers who have taught at the primary school, high school, college and doctoral levels with extensive experience in the field of cognition, The information gathered was examined using analytical induction. The findings reveal that primary education teachers design and perform classroom activities using tools and strategies to develop Reading Comprehension at different points in time as an implicit objective. This reflects that DI can be used as a primary tool to enhance Reading Comprehension. Yet based on the research, to garner optimal results, teachers must be informed and well-trained in Instructional Design to make a significant difference in ensuring student Reading Comprehension success.

KEYWORDS: Comprehension, Reading, Design, Instruction, Cognition. 


\section{INTRODUCCIÓN}

La comprensión lectora es una actividad de alta complejidad que puede alcanzar diversos niveles de complejidad, esto dependiendo de diversos factores que pueden ser ambientales, personales o del propio texto; de hecho, la comprensión lectora es más bien una destreza o un conjunto de ellas y no precisamente un cuerpo de conocimientos, hechos o conceptos. La lectura conlleva a la compleja interacción de procesos (perceptivos, cognitivos y lingüísticos) los cuales son mediados por aspectos como la experiencia o los conocimientos previos, tanto como por aquellos objetivos planteados para la lectura (Elosúa y García, 1993).

Actualmente, la comprensión lectora y su desarrollo a un nivel más profundo ha cobrado importancia, docentes de diferentes niveles educativos manifiestan que ante las nuevas exigencias del entorno social, político y económico de manera recurrente, la necesidad imprescindible de que el alumnado no únicamente extraiga información, sino que logre analizarla, manipularla, adecuarla, sistematizarla e incorporarla en las estructuras cognitivas para hacer un uso eficiente de dicha información.

La educación primaria en México forma parte del nivel de Educación Básica, donde se plantea una serie de propósitos curriculares relacionados implícitamente al desarrollo de la comprensión lectora del estudiantado en este nivel educativo. Concretamente, el plan de estudios publicado en 2011 señala que en la Educación Básica se busca que el alumnado aprenda y desarrolle habilidades para hablar, escuchar e interactuar, además de comprender, interpretar y producir diversos tipos de textos, a transformarlos y crear nuevos géneros y formatos; es decir, reflexionar individualmente o en colectivo acerca de ideas y textos (Secretaría de Educación Pública [SEP], 2011).

Pero para que el alumnado consiga aprender y desarrollar dichas actividades es necesario que el/la docente desarrolle un diseño minucioso de actividades de aprendizaje, de tal forma que el propio plan de estudios demanda el seleccionar estrategias didácticas que favorezcan la movilización de saberes, además de reconocer que los referentes para su diseño son los aprendizajes esperados, señalados en el programa de estudios, y que las actividades generen ambientes de aprendizaje colaborativo que favorezcan experiencias significativas al considerar evidencias de desempeño que brinden información al profesorado para la toma de decisiones y continuar impulsando el aprendizaje del estudiantado (SEP, 2011).

Ello hace referencia a ciertos elementos pertenecientes a la teoría del Diseño Instruccional (DI), en la que Reigeluth (2013), señala que los roles para docentes incluyen el ser un diseñador del trabajo del alumnado, facilitador del proceso de aprendizaje y mentor atento, además que la instrucción para el aprendizaje implica una amplia variedad de herramientas, tanto para el espacio de la tarea como para el espacio instruccional. En ese sentido, se plantea la necesidad de comprender la forma en la cual el personal docente efectúa el DI para desarrollar la comprensión lectora en el alumnado de educación primaria, con un par de casos de docentes que compartieran sus experiencias y formas de actuar.

Queda expuesta entonces una cuestión central, que se ha podido evidenciar desde la experiencia, tanto en investigación como en el ejercicio de la docencia, y es que el cuerpo docente siente el compromiso de desarrollar un nivel profundo de comprensión lectora en sus estudiantes, pero la manera en que lo hacen, cómo se preparan, qué herramientas usan, qué estrategias diseñan, no está del todo claro, una vez que se tiene claridad sobre los qué, cómo y para qué el beneficio será tangible tanto para el profesorado como para sus estudiantes.

Por todo lo anterior, la presente investigación que se ha fijado el objetivo de analizar de qué manera el personal docente diseña su instrucción pensando en las cuestiones como los objetivos, valores, métodos, herramientas y actividades que desarrollan la comprensión lectora en sus estudiantes, para esto se establecen en un primer momento, los antecedentes que configuran estas variables como una preocupación actual y de suma importancia, sus hallazgos, sus constructos y aportaciones para el campo tanto del DI y de la comprensión lectora. 
En el siguiente espacio se acotan los referentes conceptuales en cuyo trabajo se cobija el análisis de los datos obtenidos; consecutivamente, se desglosan las decisiones y procesos metodológicos que rigen la investigación y que permiten, posteriormente, discutir lo que esos datos representan a la luz de la teoría y la experiencia y finalmente llegar a las ideas y constructos concluyentes que dan un cierre al proceso de investigación desarrollado, pero que, al mismo tiempo, abren un amplio abanico de posibilidades para posteriores investigaciones.

\section{ESTADO DE LA CUESTIÓN}

Al ser la lectura una de las tareas cotidianas de mayor impacto en la vida de una persona, se torna sustancial que los espacios educativos centren sus esfuerzos en la construcción de diseños que la promuevan eficazmente, por tal motivo, no sorprende la gran cantidad de investigaciones que bien de forma individual o de forma vinculada, dan cuenta de que las variables de investigación son de importancia capital.

En relación con el diseño instruccional, Zermeño, Guzmán y Arroyo (2014), demostraron que, de hecho, para el estudiantado este aspecto sí es importante, pues estos perciben no solo la pertinencia de las estrategias utilizadas o el perfil de quien lo imparte, sino que además consideran la opción de la impartición combinada, es decir tanto presencial como mediada por la tecnología como la más viable y enriquecedora.

Coincidentemente, Eraso, Vela, Calderón y Sánchez (2017), sostienen a partir de su intervención, que el diseño instruccional basado en modelos híbridos que combinan Ambientes Virtuales de Aprendizaje (AVAS) y cursos presenciales permiten al/la docente hacer efectivo el proceso de aprendizaje, sirviendo como una guía que oriente la planificación adecuada para crear un ambiente de aprendizaje orientado al logro de objetivos donde estudiantes, docentes, los recursos, el ambiente y todos los elementos adyacentes actúen en apoyo de ese fin.

Otras investigaciones arrojan luz sobre cómo el entrenamiento a partir del diseño, para la mejora de habilidades de comprensión, son una herramienta promisoria, por ejemplo, Lan, Lo y Hsu, (2014) y Hassan (2017) dan cuenta de cómo a partir de un entrenamiento adecuado el acopio y la conciencia de estrategias metacognitivas garantiza una mejora en la comprensión de lo que el estudiantado lee, del mismo modo Dabarera, Renandya y Zhang (2014) corroboraron que existe una relación entre la conciencia metacognitiva y la mejora de la comprensión lectora. Además, la instrucción en estrategias resulta efectiva para aumentar la conciencia metacognitiva, y se relaciona con incremento de comprensión de la lectura. Esta significatividad encontrada entre el entrenamiento y la comprensión lectora es una vez más demostrada por Tavakoli, (2014), quien, como resultado de su investigación experimental, probó una fuerte correlación positiva entre el conocimiento metacognitivo informado de las estrategias de lectura y el rendimiento de comprensión lectora.

En su tesis de grado, Castro (2019) desarrolla un diseño instruccional con estudiantes de 3er grado de secundaria, basado en el enfoque estratégico de la comprensión lectora, sus resultados demuestran que efectivamente, el diseño instruccional es el factor que determina el progreso del alumnado respecto de esta habilidad en contraste con investigaciones como las de Madero y Gómez (2013); Farías y Araya (2014); Paba y González (2014); Pérez y La Cruz (2014) y Fernández y Sanz (2016) convergen en la idea de que las habilidades lectoras del estudiantado no son desarrolladas en su potencialidad, menos aún si se habla de las habilidades metacognitivas, iniciando por el hecho de que en la mayoría de las prácticas de lectura no se tiene la atención participante del alumnado.

Queda en evidencia pues, que el vínculo entre el diseño de la instrucción y el desarrollo o mejora de la comprensión lectora es fuerte y se encuentra sellado por la creciente necesidad de individuos diestros en la búsqueda, selección y comprensión de la vorágine de información escrita a la que están expuestos. 


\section{REFERENTES CONCEPTUALES}

Gómez, Vila, García, Contreras y Elosúa (2014) sostienen la importancia del sistema de memoria de trabajo en la comprensión de lectura desde modelos clásicos de comprensión de textos. De acuerdo con sus hallazgos, la comprensión es a menudo un proceso complejo que es logrado dentro de la memoria de trabajo y requiere una gran cantidad de recursos cognitivos y metacognitivos, incluido el almacenamiento y eficacia de procesamiento; por ello, la comprensión lectora es una tarea en la que los procesos ejecutivos están muy implicados.

Al leer ocurre una interacción sumamente compleja entre procesos perceptivos, cognitivos y lingüísticos, que a su vez son mediados por la experiencia y los conocimientos previos que posee el lector igual que por los objetivos de la lectura y las características propias del texto.

Elosúa y García (1993) admiten que para lograr una lectura comprensiva se deben considerar, en un primer momento, los niveles que van de la decodificación, la extracción literal, la comprensión inferencial, hasta la metacomprensión. En seguida, considerar los tres momentos de la tarea de lectura, la planificación, el monitoreo y la supervisión, a través de las que se llega no solo a la mera comprensión, sino a la metacomprensión y, finalmente, observar las estrategias cognitivas que utiliza el lector, que pueden ser de focalización, de organización, de elaboración o de resolución de problemas.

Cuando un/a docente comprende que decodificar o comprender, planificar o evaluar, son procesos importantes pero que no son los únicos relevantes durante la lectura, tiene más posibilidades de intervenir adecuadamente para ayudar al lector a resolver las dificultades que se presentan a sus estudiantes durante la lectura.

En todo esto el DI es fundamental, porque asegura la toma de decisiones cuidadosamente elaboradas y que fortalezcan el desarrollo de la comprensión de forma sistemática y consciente, en este rubro Perkins (1995) asegura que el alumnado aprende más cuando tiene una oportunidad razonable y una motivación para hacerlo, con ello plantea que las condiciones para el aprendizaje implican el presentar información clara, donde se describan los objetivos y conocimientos requeridos, además de prácticas reflexivas donde haya oportunidades de ocuparse activa y reflexivamente de aquello que deba aprender, una realimentación informativa y una fuerte motivación intrínseca y extrínseca.

Otro exponente importante para el DI sistemático es Gagné (1987), quien establece las condiciones necesarias para que un diseño logre aprendizaje en sus destinatarios, las divide en dos grupos, las primeras son internas al individuo y las explica en nueve eventos, basándose en la interacción medio - receptor, el cual activa el proceso de aprendizaje, estimulando los receptores del sujeto y permitiéndole captar y seleccionar la información. Las otras son externas al estudiantado y las define como aquellos eventos de instrucción que permiten que se produzca un proceso de aprendizaje. Estas condiciones pueden entenderse como la acción que ejerce el medio sobre el sujeto.

Entonces, el DI es una tarea que debe enfocarse en generar los estímulos necesarios para que las condiciones internas y externas generen aprendizajes. Al ser la lectura una tarea estratégica, que obliga a mantener un acervo amplio disponible en la memoria, un DI bajo el enfoque cognitivo conductual de Gagné (1987) es una opción por considerar.

\section{Procedimientos metodológicos}

La investigación cualitativa es, en palabras de Sandín (2003), una actividad sistemática orientada a comprender en profundidad fenómenos educativos y sociales, a la transformación de prácticas y escenarios socioeducativos, a la toma de decisiones y al descubrimiento y desarrollo de un cuerpo organizado de conocimientos. 
De un estudio de casos se espera que abarque la complejidad de un caso particular, se estudia un caso cuando tiene un interés muy especial en sí mismo. Se busca el detalle de la interacción con sus contextos. El estudio de casos es el estudio de la particularidad y de la complejidad de un caso singular, para llegar a comprender su actividad en circunstancias importantes (Stake, 1998)

Latorre, Del Rincón y Arnal (1996) identifican varias ventajas del uso de este tipo de estudios, por ejemplo: es útil en investigaciones a pequeña escala, está abierto a retomar otras condiciones personales o institucionales diferentes, pero, sobre todo, lleva a tomar decisiones, a implicarse a desenmascarar prejuicios y preconcepciones.

En la presente investigación se utilizó como técnica de recolección de datos la entrevista semiestructurada, una a cada docente, se eligió porque puede ser un instrumento valioso para obtener información; sin embargo, para que sea exitosa debe reunir requisitos como establecer el rapport, desarrollarse en un clima de tranquilidad, amabilidad y confianza entre otros (Münch y Ángeles, 2003).

Se solicitó a cada uno de los dos docentes su consentimiento para grabar el audio de la entrevista, en caso de que algún dato importante pudiera perderse; ambos accedieron. Aunado a esto, se tomó nota de las respuestas, así como de las reacciones de los entrevistados. Una vez obtenidos los datos de cada entrevistado, se transcribieron las entrevistas y se procedió al análisis de la información.

Dicho análisis se organizó siguiendo el procedimiento denominado inducción analítica (Goetz y LeCompte, 1988). Este procedimiento implica el examen de los datos en busca de categorías de fenómenos y de relaciones entre ellas que puedan explicar el fenómeno estudiado; para la presente investigación se llevaban como categorías generales el diseño instruccional y la comprensión lectora. Sin embargo, durante el análisis se permitió que fueran los datos los que construyeran categorías o subcategorías, según fuera el caso. El rigor metodológico (Ruiz, 1999) que da validez, credibilidad y confirmabilidad a los resultados, se obtuvo mediante la confirmación con el participante y la triangulación teórica.

Todo el proceso anterior se desarrolla en función del propósito de la investigación, que busca reconocer las prácticas del cuerpo docente dentro de un discurso, integrarlas en categorías que permitan vislumbrar el tipo de diseño al cual el profesorado recurre para desarrollar la comprensión lectora en sus estudiantes.

Los participantes, a quienes se denominará en lo sucesivo E1 y E2, cursaron la licenciatura en Educación Primaria, la maestría en Pedagogía y cuentan con el grado de doctor. Son docentes de educación primaria y a medio tiempo en una institución de Educación Superior. Además, imparten también cátedras, cursos, diplomados y capacitaciones en diversas instituciones públicas y privadas del Estado. Ambos están en capacitación constante, así como fuertemente involucrados en el área del Diseño de la Instrucción y la cognición donde han producido numerosos diseños exitosos para instancias públicas y privadas.

Las razones que motivan la elección metodológica se basan en que, por experiencia con investigaciones previas el profesorado responde con mayor libertad debido a su cercanía y familiaridad, a metodologías cualitativas, que propician el rapport, generando un clima de confianza donde sus opiniones, sensaciones y percepciones son tan importantes como la teoría o la estadística.

\section{ANÁLISIS Y DISCUSIÓN DE RESULTADOS}

Como recién se dijo, lo que el profesorado tiene que decir al respecto de sus prácticas, sus decisiones o sus necesidades es un elemento sustancial para comprender el funcionamiento del sistema educativo, para configurarlo y estructurarlo de acuerdo con principios centrados no solo en las políticas educativas, sino en el acto propio de la enseñanza-aprendizaje. 


\section{La comprensión lectora. Una habilidad para la vida}

Leer es una tarea que ejecutan tanto el personal como el alumnado y ambos se ven en la necesidad de comprender para aprender, Vallés (2005) considera que la lectura mantiene una relación directa con la comprensión, y tiene una relevancia que va más allá del terreno de la educación, pues está configurada como una herramienta toral para llegar al conocimiento y lograr construir la realidad, presentándose como una competencia sustancial en el procesamiento de la información y que genera a su vez otras competencias tales como el análisis, la imaginación, la deducción, la lectura de códigos, símbolos, entre otras.

Esta perspectiva está en consonancia directa con lo que el cuerpo docente vive cotidianamente en sus aulas y en su vida personal; la experiencia les da la certeza de que la lectura comprehensiva no se acota a la normatividad o lo institucional: “... la comprensión lectora va más allá de las fronteras institucionales más allá de los planes y programas que se puedan generar, en la escuela o en un nivel educativo" (N. Vergara, comunicación personal 22 de septiembre, 2018)

“... el maestro debe aplicar técnicas de recordar, intuir, criticar de forma cotidiana, no solo en un momento, debe ser continuo hay que generar espacios para que el alumno lea, opine, no solo cumplir con la normatividad" (D. Rodríguez, comunicación personal 12 de noviembre, 2018)

Se deja entrever que quizá la comprensión lectora ha sido reducida ocasionalmente a los aspectos que si bien la fortalecen no la garantizan, como la velocidad, la fluidez o la entonación y los programas de estudio anteriores y actuales proponen una serie de estrategias que a la luz de las reformas quizá podrían parecer obsoletas, el profesorado aún valora estos aspectos y estas herramientas, pero les asignan un papel complementario.

... se debe utilizar lo que hace mucho tiempo en los programas de estudio del 94 denominábamos estrategias de lectura ... utilizarlas porque a mi parecer siguen siendo vigentes, aunque no los programas pues si en la teoría pedagógica nos pueden servir de mucho (N. Vergara, comunicación personal 22 de septiembre, 2018)

Esta afirmación hecha por el docente se apega a la intención con que dichos materiales fueron elaborados, los ficheros de actividades, el libro para el docente y el Programa Nacional de Lectura y Escritura (Pronales) se adaptaron a los intereses, necesidades y dificultades de las niñas y los niños, y constituyen referentes para que el profesorado diseñe las actividades didácticas en función de la atención tales necesidades y dificultades (Bastián, 2006).

... antes se le daba más importancia... hace años se desarrollaba además la velocidad por la comprensión, ahora ni uno ni otro, yo hice un estudio de la velocidad y su relación con la comprensión, y si sucede que se requiere un mínimo de velocidad para lograr la comprensión (D. Rodríguez, comunicación personal 12 de noviembre, 2018)

Si bien los enfoques actuales han dejado de lado la relevancia de la velocidad lectora como elemento de la comprensión, el cuerpo docente aún considera que debe fomentarse no como criterio de calidad, sino como factor de mejora. Esto tiene una directa concordancia con lo que afirman Ballesteros, Calero, López, Jiménez, Ribeiro, Santos y Villanueva, (2013), que el automatismo determinado por la velocidad es necesario en las etapas iniciales del aprendizaje de la lectura, pues los lectores lentos tienden a perder el interés y eventualmente dejan de leer.

Se evidencia pues, que la comprensión lectora es un complejo entramado de capacidades que van desde lo mecánico hasta lo cognitivo, que se involucran más allá de lo meramente institucional en la vida de un individuo y que la escuela debe buscar y generar no solo espacios, sino un estilo de vida donde el alumnado se inserte como sujetos que comprenden, aprenden y generan cambios. A esto cabe una reflexión de Lerner (2001):

Lo necesario es hacer de la escuela una comunidad de lectores que acuden a los textos buscando respuesta para los problemas que necesitan resolver, tratando de encontrar información para comprender mejor algún 
aspecto del mundo que es objeto de sus preocupaciones, buscando argumentos para defender una posición con la que están comprometidos o para rebatir otra que consideran peligrosa o injusta (p. 1)

Completaría esta afirmación tan profunda con el comentario que hace uno de los docentes al respecto de cuán importante es para el desarrollo de un individuo crítico y propositivo el hacer una lectura de comprensión dado el momento histórico y social que ha tocado en suerte vivir, “... sirve a lo largo de la vida, más en la época posmoderna, es una herramienta muy necesaria porque estamos invadidos de información así que necesitamos seleccionar lo que es importante y lo que no" (D. Rodríguez, comunicación personal 12 de noviembre, 2018)

\section{El papel del profesorado para el desarrollo de la comprensión lectora en sus estudiantes}

Qué utilidad tendría la conciencia en el profesorado sobre la relevancia de la comprensión lectora como herramienta para la vida que en la mayoría de los casos se adquiere y perfecciona en la escuela, si no llegan a asumirse como actores responsables de su desarrollo y fortalecimiento. Conviene entonces reflexionar qué tanto saben del rol que desempeña el personal docente en todo este proceso, de qué manera favorecen el desarrollo de una comprensión profunda, cómo se presentan ante sus estudiantes para ser modelos y facilitadores.

Resulta interesante la forma en la cual el profesorado se preocupa por la forma en que se acerca a sus estudiantes, busca, indaga, son empáticos con las características y gustos de sus estudiantes lo que de acuerdo con su percepción facilita que las niñas y los niños desarrollen gusto por la lectura y tengan como consecuencia un acto comprensivo de ella "hay que situarse en el lugar del alumnado y pensar qué es lo que les interesa aprender qué es lo que les gusta y hacer un poquito de esfuerzo de buscar o encontrar esos personajes que a ellos les gustan" (N. Vergara, comunicación personal 22 de septiembre, 2018).

“...yo les recomiendo a los padres que les compren libros de interés... para que se motiven a leer primero y que vean qué les sirve leer, deben también verte leyendo, que vean los beneficios” (D. Rodríguez, comunicación personal 12 de noviembre, 2018).

En coincidencia con las ideas de los entrevistados, Viana, Ribiero y Santos (2014) aseveran que debe tenerse un especial cuidado con los textos que se seleccionan pues quizá no sean punto de llegada, sino un punto de partida para la lectura, además conocer los intereses de las niñas y los niños es un paso importante para que la selección del material de lectura ofrezca potencialidades en términos de motivación para la lectura.

Pero no solo la atención a los intereses del alumnado o la selección del material pertinente es lo que ocupa al profesorado, pues tienen claro que para fomentar una lectura de comprensión deben ser ellos los primeros en tener dicha habilidad, por lo tanto, su preparación se orienta también al ámbito personal.

... la comprensión lectora tiene muchas aristas que me parece importante tomar como referencia, de entrada, hay que prepararse en varios sentidos respecto a la comprensión lectora, primero en el sentido personal de lo que yo comprendo cuando leo y en el sentido de maestro (N. Vergara, comunicación personal 22 de septiembre, 2018).

Aunado a esto, saben que en muchas de las ocasiones la responsabilidad de lograr que sus estudiantes se adentren en la lectura, desarrollen gusto por ella y perfeccionen sus habilidades lectoras como la metacomprensión dependerá totalmente de ellos, pues ya sea en atención a contextos desfavorables o situaciones familiares complejas, deberán ser más que solo un apoyo escolar para el alumnado.

... se debe saber qué probabilidades le ofrece el contexto ... qué apoyo por parte de los padres de familia, o sea no puedo llegar encargando tareas si sé que no son apoyados de verdad por los padres de familia entonces tenemos que conocer pues prácticamente todo acerca del contexto cual es el ambiente familiar, el comunitario (D. Rodríguez, comunicación personal 12 de noviembre, 2018).

Hasta este punto se denota un claro compromiso del profesorado por entregar lo mejor de sí mismos a su tarea, prepararse, asumirse como responsables de un preciado tesoro, la mente fértil de sus estudiantes, 
con la conciencia de que el buen lector, requiere de buenos textos, pero sobre todo de buenos modelos. Como sugiere Day (2006), un cuerpo docente apasionado se muestra comprometido, entusiasta e intelectual y emocionalmente entusiasmado con su trabajo, es consciente de los desafíos de contextos sociales, tiene un sentido claro de identidad y cree que puede favorecer el aprendizaje y el rendimiento de todos sus estudiantes.

\section{Diseñar para comprender lo que se lee. Las estrategias, herramientas y metodologías para la comprensión lectora}

Aún con la certeza de un compromiso por parte del profesorado y un denotado esfuerzo por prepararse y dar lo mejor, desarrollar la comprensión lectora no es tarea sencilla, exige premeditación, sistematización y cuidar el diseño de las actividades; al respecto Silas y Gómez (2013) pudieron demostrar que el alumnado con docentes que se muestran más proclives a introducir cambios en su práctica para mejorar su comprensión obtuvieron un logro mayor.

Es factible afirmar entonces que el personal docente es absolutamente consciente de que la comprensión lectora a un nivel más profundo exige preparación, demanda en un primer momento conocer a los sujetos que se va a entrenar. Es consciente también de lo importante que resultan factores que acercan al alumnado a la lectura como práctica de vida, cuestiones contextuales, culturales, escolares, son algunas de las que identifican como medulares para el desarrollo de esta habilidad en sus estudiantes.

... bueno son varios factores que considero yo muy importantes que el docente tome en cuenta, en el grupo de entrada las características generales del alumnado por ejemplo características de aprendizaje, de desarrollo, familiares, culturales, emocionales (N. Vergara, comunicación personal 22 de septiembre, 2018).

... se debe conocer pues prácticamente todo acerca del contexto, cuál es el ambiente familiar, el comunitario, el escolar, con qué recursos cuento dentro de la escuela y pues ya algunas cuestiones muy específicas del alumnado y el historial académico que me va a permitir tomar decisiones (D. Rodríguez, comunicación personal 12 de noviembre, 2018).

Aquí queda clara la primera parte que demanda el diseño, especialmente cuando va dirigido a espacios institucionales donde se es responsable de un grupo por largos periodos: El diagnóstico, que según Alfonzo (2003) es una actividad esencial para la planificación eficaz de la instrucción, así como para evidenciar los cambios producto del aprendizaje adquirido por el estudiantado.

Ahora bien, aunado a ese diagnóstico y con base en la teoría instruccional son varios elementos que se deben considerar para diseñar el trabajo por desarrollar, el profesorado no es ajeno, pues como se mencionó posee un amplio conocimiento al respecto, por este motivo comienza sus diseños contemplando los objetivos por lograr: “... el tipo de objetivos tendrían que ver con el tipo de conciencia, qué conciencia en cuanto a comprensión quiero desarrollar con mis alumnos" (N. Vergara, comunicación personal 22 de septiembre, 2018).

“... los objetivos acompañan el seguimiento del currículum, o sea no dices voy a dejar el currículum si no aprovecho lo que el currículum me da para trabajar la comprensión lectora siempre y cuando te hayas fijado esa meta" (D. Rodríguez, comunicación personal 12 de noviembre, 2018).

Es importante concebir estos objetivos como aquello que se quiere alcanzar con el alumnado, pues son quienes deberán llegar a esa meta (Hernández, 1989). Estos objetivos guiarán la selección de la metodología más acertada según la meta y las necesidades detectadas en el diagnóstico.

“... los métodos tienen una directa asociación entre las formas en que aprendemos a leer y a escribir con las formas en que posteriormente aprendemos a comprender y a interpretar los textos” (N. Vergara, comunicación personal 22 de septiembre, 2018).

“... los métodos van en relación qué es lo que hace falta a cuál es el siguiente nivel” (D. Rodríguez, comunicación personal 12 de noviembre, 2018). 
Una vez que consideran este aspecto tan importante, se está en posibilidad de diseñar actividades o estrategias pertinentes para alcanzar los objetivos con la metodología seleccionada, más aún cuando ese objetivo es alcanzar el máximo nivel de comprensión, la metacognición.

Con lo anterior perfectamente claro, se debe pues asegurar que las tareas de comprensión lectora se enfoquen en ejercicios encaminados tanto a la eficacia como a la eficiencia en los procesos involucrados (decodificación y significado de las palabras) al tiempo que se dirigen a integrar estrategias de autorregulación para que el estudiantado pueda desarrollar una mayor conciencia y control de sus propios procesos durante la comprensión lectora (Orbea y Villabeita, 2010).

“... bueno son una multiplicidad de estrategias por cada uno de los esquemas o de los niveles de comprensión en los cuales estén el alumnado y en función de eso se diseñan” (N. Vergara, comunicación personal 22 de septiembre, 2018).

“... las estrategias se encaminan a establecer una relación, entre el texto que leyeron y la realidad de ellos y puedan no sé más adelante relacionarlas como con qué más conocen, qué más han leído” (D. Rodríguez, comunicación personal 12 de noviembre, 2018).

Existen además otros elementos fundamentales al diseñar la instrucción, las herramientas o recursos, tanto para desarrollar el trabajo o actividad como para la evaluación. Hernández (1989) categoriza que elegir los medios adecuados supone saberlos integrar en las propias teorías instruccionales, saberlos relacionar con los objetivos o fines educativos que se persiguen, saberlos utilizar de acuerdo con las distintas condiciones previstas en la situación de enseñanza-aprendizaje. Al cuestionarlos sobre los medios o recursos necesarios para su trabajo comentaron:

... pues primero libros verdad, este, pero no nada más libros, porque a los niños ya no les gusta leer nada más libros hay muchas cosas que les gusta, les gusta mucho la parte tecnológica eeh y hacer lectura de manera o con recursos tecnológicos. (N. Vergara, comunicación personal 22 de septiembre, 2018).

... que sean textos que les interesen porque luego los obligamos a leer cosas que no les interesan y por eso no comprenden porque no les interesa. Yo les pido textos que traen de sus casas, la mayoría los descargan de internet, y llevan textos de robots, de tecnología, de fútbol de baile, etc. (D. Rodríguez, comunicación personal 12 de noviembre, 2018).

El aspecto que puede considerarse final, pero que es al mismo tiempo el reinicio del proceso es la evaluación, y al respecto se identifican dos vertientes, la primera coincide con Hernández (1989) en el sentido de ver la evaluación como el elemento del diseño que aporta información de lo sucedido en el proceso enseñanzaaprendizaje, permite la comprobación de los objetivos y la metodología, pero, sobre todo, actúa como factor de retroalimentación para estudiante y docente.

... la evaluación como actividad debe estar muy contextualizada a las características de tus alumnos...habrá que realizar ciertas adaptaciones para que sea muy pertinente con lo que se está trabajando con ellos y bueno el proceso siempre tiene que ver con distinguir qué estamos evaluando en dado momento no, o qué tipo de propiedad de la comprensión lectora estamos evaluando, en ese sentido pues serán los instrumentos o las modalidades para la evaluación (N. Vergara, comunicación personal 22 de septiembre, 2018).

La otra perspectiva es instrumental, aunque no por eso menos valiosa, porque la comprensión lectora pertenece a las actividades orientadas a la práctica (Reigeluth, 2013) y debe tener una evidencia medible, para lo cual se vuelve necesario poseer un instrumento que ratifique lo observado.

“...yo utilizo el instrumento oficial, el que se nos proporciona para evaluarla... lo que buscamos es si el alumno recuerda el contenido, establece relaciones, hace inferencias o predicciones, y así vemos el nivel de avance bimestralmente" (D. Rodríguez, comunicación personal 12 de noviembre, 2018).

Ante un escenario donde el personal docente deposita su confianza en los elementos brindados por la autoridad educativa (SEP, 2011), entiéndanse los instrumentos para evaluar, resulta pues fundamental que los antes mencionados tomen muy en serio su tarea al diseñarlos, deben seguir los pasos pertinentes para validarlos y que sean una verdadera herramienta útil, tanto para estudiantes como para el profesorado. 
Dentro de estos pasos o pautas a seguir, se identifica que el grupo docente se acerca a la propuesta de Robert Gagné (1987), porque consideran aspectos tanto internos como externos al alumnado, tratan de diseñar actividades motivantes, novedosas, compartir sus propósitos con sus estudiantes, desarrollar valores a partir de la actividad lectora, en fin, buscan generar la condiciones para que el aprendizaje pueda suceder.

A este punto de la reflexión se puede identificar la enorme concordancia entre los aspectos que toma en cuenta el profesorado para lograr una comprensión en sus estudiantes y aquellos de la teoría instruccional, con esto pretenden fortalecer su práctica y medir, de manera más efectiva, el logro del aprendizaje alcanzado, así como la profundidad con que la comprensión lectora ocurre en el proceso de sus estudiantes.

\section{La metacognición en la lectura, una meta posible y obligada}

Consecuentemente con dicha reflexión al respecto de cómo el cuerpo docente se prepara a partir de un diseño instruccional y así desarrollar la comprensión lectora, se detectó en los docentes entrevistados una clara tendencia a buscar, diseñar y propiciar momentos y actividades que fomenten en sus estudiantes una comprensión lectora que no se limite a la decodificación y la extracción literal, sino que llegue al último peldaño, la metacomprensión del texto.

“... buscar cómo propiciar que mis alumnos vayan más allá del hecho literal que está plasmado en el escrito” (N. Vergara, comunicación personal 22 de septiembre, 2018).

“... al momento de leer textos no te quedes en esa parte nada mas de recuperar, sino que hay que establecer actividades para lograr el siguiente nivel” (D. Rodríguez, comunicación personal 12 de noviembre, 2018).

Existen diversas clasificaciones para los niveles de comprensión lectora, en este caso se diferencian en tres que irían desde un nivel más superficial a un nivel de mayor profundidad (Elosúa y García, 1993):

1. Decodificar. Como su nombre lo indica, se refiere a descifrar un código; el objetivo es poder dar un significado a las letras impresas.

2. La comprensión literal se refiere a lograr combinar el significado de varias palabras apropiadamente para formar proposiciones.

3. La metacomprensión es el nivel óptimo y hace referencia a la conciencia y control que el lector tiene de su proceso de comprensión. Implica el establecimiento de metas, monitorear su progreso, insertar modificaciones necesarias y evaluar el resultado.

El profesorado reconoce estos niveles y trabaja, en consecuencia sabe que la comprensión lectora debe ser un proceso interno y personal en un primer momento para luego trasladarlo al trabajo con sus estudiantes:

... la comprensión lectora tiene varios niveles o tiene una estructura definida donde yo puedo apoyar a una parte o a otra puedo jerarquizar que se logre una cosa primero y luego a partir de eso logramos otra de mayor jerarquía (D. Rodríguez, comunicación personal 12 de noviembre, 2018).

Saben además que deben tener en cuenta cuestiones como la edad, el nivel educativo, la temática abordada y las estrategias para propiciar el que sus estudiantes progresen de un nivel a otro:

... cuando hablo en los primeros grados con una recuperación más textual que inferencial del contenido del texto... paulatinamente el ejercicio, el cuestionamiento tiene que irse complejizando de tal manera que puedan responder con la idea que está ahí más no con las palabras explícitas que establece la lectura (N. Vergara, comunicación personal 22 de septiembre, 2018).

Es posible notar el interés y conocimiento del profesorado al respecto del proceso que deben seguir sus estudiantes para alcanzar la metacomprensión, buscan las maneras de apoyar este desarrollo de forma natural y fluida, comenzando por atender su propio desarrollo como lectores. Cassany (2001) afirma que resulta más fácil aprender a cocinar si se es asistente de un gran chef que leyendo libros de recetas o ensayando solo por la cuenta de cada uno; el/la docente es el mejor experto que el aprendiz encuentra en el aula y por lo tanto la forma en que asuma y se posicione en su función podrá favorecer y potenciar las habilidades de sus estudiantes. 
... me gusta leer con mis alumnos novelas, textos científicos, leer periódicos y hay un ejercicio interesante al respecto cuando nos ponemos en un momento inicial a subrayar ideas principales del texto que nos ayuden a comprender la idea esencial, pasamos por varios momentos podría yo hablar de momentos ascendentes y momentos descendentes dependiendo de la lógica que sigamos en esta interpretación del texto (N. Vergara, comunicación personal 22 de septiembre, 2018).

... por ejemplo si es en historia, causas consecuencias, qué resultados trajo, cómo logramos hoy en día lo que ha sucedido no le hace que estemos hablando de la prehistoria o escríbeles una carta a los personajes no le hace que no la van a poder leer, hace poco les decía escríbele una carta a Lucy de un cráneo que se encontró hace más de 3.5 millones de años (D. Rodríguez, comunicación personal 12 de noviembre, 2018).

Las estrategias para desarrollar una metacomprensión buscan siempre la conciencia del alumnado sobre sus procesos cognitivos para poder así seleccionar la forma más adecuada de afrontar el texto. Es fundamental, como manifestaron los docentes, que propicien el gusto y la satisfacción por la lectura mientras logran además que sus estudiantes hagan lecturas más analíticas y profundas valiéndose de numerosas estrategias. En el caso de E1, por ejemplo, de focalización y en el de E2, de un diálogo con los textos.

De forma inconsciente, ambos docentes dejan implícito al que se considera el fin último de la metacomprensión, el cual es desarrollar la capacidad de comunicarse con el texto, construir y reconstruir el mensaje, organizar y reorganizar la información, ser capaz de extraer de todo un libro un enunciado que lo explique, ante esta responsabilidad el profesorado siempre busca que sus estudiantes se sumerjan un poco más:

... será pues la habilidad del maestro para hacer lo más certeras de interpretaciones posibles de acuerdo con los datos que están por ahí ... vienen lo elementos críticos ... debemos preocuparnos por eso del lado de nuestros alumnos ¿no? De que interpreten la información ...que se cuestionen qué tan cierto o qué tan falso, qué tan neutral puede ser el posicionamiento del autor al generar ese escrito que ellos están leyendo, que establezcan propuestas de cómo podrían hacer las cosas mejor a partir de eso que están por ahí leyendo que procuren generar cambios que se dan desde la lectura (N. Vergara, comunicación personal 22 de septiembre, 2018).

También comprenden que deben estimular a sus estudiantes para llegar a

... niveles cognitivos cada vez más altos, aquí no se trata de conocer sino de comprender y podemos ir más allá, podemos analizar, podemos evaluar qué está haciendo bien el personaje, qué hubieras hecho tú, qué otro final le hubieras dado, entonces siempre que hay lectura pues tenemos la oportunidad de trabajar la comprensión lectora... si yo quiero que mis alumnos hagan inferencias entonces las actividades van a estar enfocadas a hacer inferencias hasta que yo empiece a notar que mis alumnos ya están haciendo inferencias y luego vamos a pasar a las comparaciones (D. Rodríguez, comunicación personal 12 de noviembre, 2018).

De estas afirmaciones se proyecta una conciencia de docentes al respecto de los procesos, esto les da más posibilidades de intervenir adecuadamente para ayudar a sus estudiantes a resolver las dificultades que se les presenten, pero se debe ser conscientes de que si se entrena a los sujetos en la práctica de ciertos procedimientos y estrategias se consigue mejorar su nivel de comprensión lectora (Elosúa y García, 1993).

\section{Conclusiones}

En la era de la información inmediata, donde la mayoría de las personas tienen acceso a fuentes inagotables de esta, se vuelve indispensable desarrollar la capacidad de leer comprensivamente, de retomar del contenido aquello genuinamente relevante, útil, verdadero y utilizar ese acervo para mejorar la vida.

Es pues, tarea del profesorado, propiciar en sus estudiantes el desarrollo de la comprensión lectora a un nivel más profundo que el acostumbrado, a partir de diseños instruccionales bien estructurados, sustentados en las teorías que explican el aprendizaje y fortalecen la enseñanza, con actividades novedosas, materiales pertinentes y objetivos realistas. 
Los tres actores principales en la comprensión lectora son el profesorado, el alumnado y el texto; la calidad de su interacción será determinante en el progreso de sus estudiantes y hasta del mismo grupo docente de un nivel a otro al dotarlo de estrategias y de la capacidad de distinguir cuál y cuándo usarlas.

La lectura es una actividad 'estratégica'. El buen lector pone en juego unos procedimientos o estrategias para obtener un resultado. Tales estrategias o destrezas son susceptibles de ser mejoradas, de convertirse en objeto del proceso de enseñanza-aprendizaje, con el fin de optimizar en los lectores su nivel de comprensión. Si la comprensión es la meta de la actividad lectora es importante que los lectores evalúen si se está logrando (Elosúa y García, 1993, p. 13)

Finalmente, todo actuar docente debe estar guiado por una intención clara, un objetivo claramente establecido, estrategias o actividades bien estructuradas, herramientas cuidadosamente seleccionadas, pero sobre todo, no debe perder de vista el nivel en el que se ubican sus estudiantes y saber a dónde quiere llegar con ellos, sin dejar de lado jamás informarles de la meta.

Esta facultad será definitivamente más eficaz en cuanto mejor y más organizado sea el diseño de la instrucción para desarrollar la comprensión en sus estudiantes, evidentemente cuando un/a docente toma la responsabilidad de formar de la manera más integral y completa posible a sus estudiantes, incorpora en su tarea diaria la consigna de diseñar el qué, cómo, cuándo, con qué, por qué y cómo comprobar de su actuación con el grupo.

Los hallazgos de la investigación arrojan luz sobre una cuestión ampliamente discutida pero poco profundizada, la necesidad de sistematizar el diseño de la instrucción capacitando al grupo docente en la tarea. Es decir, que los cursos, talleres o consejos donde se reúne al profesorado sean espacios de preparación y crecimiento, de consenso y creación, para que se hagan de herramientas y recursos cada vez más efectivos con los que diseñar estrategias que no solo desarrollen, sino que perfeccionen la comprensión de texto en el alumnado.

\section{Aportaciones de la investigación}

TABLA 1

Estrategias propuestas por el profesorado a tomar en cuenta en el DI para la comprensión lectora

\begin{tabular}{|c|c|c|}
\hline Estrategias Docente & Para profundizar en el nivel de comprensión & Para fomentar la lectura \\
\hline E1 & $\begin{array}{l}\text { Diseñar las actividades en función del nivel que se desea } \\
\text { alcanzar . Incorporar la tecnologia a las tareas de lectura } \\
\text { Utilizar la evaluación como herramienta de } \\
\text { retroalimentación. Retar al alumnado a que lleguen a } \\
\text { niveles cada vez más altos de complejidad en las actividades }\end{array}$ & $\begin{array}{l}\text { Demostrar interés en los intereses del } \\
\text { alumnado conocer a fondo las } \\
\text { características de aprendizaje, de desarrollo, } \\
\text { familiares, culturales, emocionales del alumnado } \\
\text { Desarrollarse primero como un buen lector }\end{array}$ \\
\hline E2 & $\begin{array}{l}\text { Recordar, intuir, criticar de forma cotidiana. } \\
\text { Vincular la lectura a la vida real del alumnado. Incorporar } \\
\text { la tecnología como recurso para la lectura. Diseñar } \\
\text { instrumentos que demuestren si el alumnado recuerda le } \\
\text { contenido, establece relaciones, hace inferencias o predicciones, } \\
\text { y así vemos el nivel de avance. Usar estrategias que } \\
\text { obliguen al alumnado a llegar más allá de la literalidad }\end{array}$ & $\begin{array}{l}\quad \text { Ser un modelo de lector } \quad \text { Propiciar el } \\
\text { apoyo de padres de familia } \quad \text { conocer todo } \\
\text { acerca del contexto, el ambiente familiar, el } \\
\text { comunitario, el escolar, con qué recursos cuento } \\
\text { dentro de la escuela y algunas cuestiones muy } \\
\text { específicas del alumnado y el historial académico }\end{array}$ \\
\hline
\end{tabular}

Fuente: Elaboración propia

Dentro de los aspectos enriquecedores de la investigación están una serie de estrategias y recursos sugeridos por los entrevistados, en la siguiente Tabla 1 se recuperan aquellos enfocados en la tarea de la lectura. 


\section{REFERENCIAS}

Alfonzo, A. (2003). Estrategias instruccionales. Venezuela: Universidad Pedagógica Experimental Libertador, Recuperado de https://bit.ly/3mZ0hD2

Ballesteros, L., Calero, A., López, R., Jiménez, E., Ribeiro, I., Santos, S. y Villanueva, J. (2013). Investigaciones sobre lectura. España: Editorial AECL.

Bastián, N. (2006). Actividades Didácticas en la Adquisición de la Lectoescritura en Alumnos de Primer Grado de Educación Primaria (Tesis de Maestría inédita). Instituto Tecnológico y de Estudios Superiores de Monterrey, Oaxaca, México.

Cassany, D. (2001). Decálogo didáctico de la enseñanza de la composición. Glosas didácticas: revista electrónica internacional de didáctica de las lenguas y sus culturas, (4), 1-3. Recuperado de https://bit.ly/36eN9Uo

Castro de la Cruz, K. (2019). Efectos de un diseño instruccional para incrementar la comprensión lectora en el curso de comunicación en alumnas del $3^{\circ}$ año de secundaria del Colegio María Parado de Bellido de Cerro de Pasco. Perú: Universidad Nacional de Educación Enrique Guzmán y Valle. Recuperado de http://repositorio.une.edu.pe/h andle/UNE/4214

Dabarera, C., Renandya, W. y Zhang, L. (2014). The impact of metacognitive scaffolding and monitoring on reading comprehension. System, 42, 462-473. doi: https://doi.org/10.1016/j.system.2013.12.020

Day, C. (2006). Pasión por enseñar: la identidad personal y profesional del docente y sus valores. España: Narcea.

Elosúa, M. R. y García, E. (1993). Estrategias para enseñar y aprender a pensar. España: Narcea.

Eraso, C. B. E, Vela, E. M. F., Calderón, S. S. E. y Sánchez, P. M H. (2017). Desarrollo de la comprensión lectora a través de un curso en línea. Un modelo ADDIE. CienciAmérica: Revista de divulgación cientifica de la Universidad Tecnológica Indoamérica, 6(3), 63-70. Recuperado de https://dialnet.unirioja.es/servlet/articulo?codigo=6163 711

Farías, M. y Araya, C. (2014). Alfabetización visual crítica y educación en lengua materna: estrategias metacognitivas en la comprensión lectora de textos multimodales. Colombian Applied Linguistics Journal, 16(1), 94-104. doi: h ttps://doi.org/10.14483/udistrital.jour.calj.2014.1.a08

Fernández, A. F., y Sanz, D. I. P. (2016). Propuesta instruccional para la comprensión lectora en alumnado con déficit cognitivo. International Journal of Developmental and Educational Psychology, 2(1), 241-248.

Gagné, R. (1987). Las condiciones del aprendizaje. México: Editorial Interamericana.

Goetz, J. y LeCompte, M. (1988). Etnografía y diseño cualitativo en investigación educativa. México: Morata.

Gómez, I., Vila, J., García, J., Contreras, A. y Elosúa, M. (2014). Comprensión lectora y procesos ejecutivos de la memoria operativa. Educational Psychology, 19, 103-111. doi: https://doi.org/10.1016/S1135-755X(13)7001 $7-4$

Hassan, F. (2017). Metacognitive strategy awareness and reading comprehension. The English Teacher, 32, 16-33 Recuperado de https://journals.melta.org.my/index.php/tet/article/view/344/234

Hernández, P. (1989). Diseñar y enseñar: teoría y técnicas de la programación y del proyecto docente. España: Narcea Ediciones.

Lan, Y., Lo, Y. y Hsu, Y. (2014). The effects of meta-cognitive instruction on students' reading comprehension in computerized reading contexts: A quantitative meta-analysis. Journal of Educational Technology \& Society, 17(4), 186-202. Recuperado de https://www.jstor.org/stable/jeductechsoci.17.4.186

Latorre, A., del Rincón, D. y Arnal, J. (1996). Bases metodológicas de la investigación educativa. España: Experiencia.

Lerner, D. (2001). Leer y escribir en la escuela: lo real, lo posible y lo necesario. México: Fondo de Cultura Económica.

Madero, I. y Gómez, L. (2013). El proceso de comprensión lectora en alumnos de tercero de secundaria. Revista mexicana de investigación educativa, 18(56), 113-139. Recuperado de http://www.scielo.org.mx/pdf/rmie/v18 n56/v18n56a6.pdf

Münch, L. y Ángeles, E., (2003). Métodos y técnicas de Investigación. México: Trillas. 
Orbea, J. y Villabeitia, E. (2010). The teaching of reading comprehension and metacomprehension strategies. A program implemented by teaching staff. Anales de Psicologia, 26(1), 112-122. doi: http://dx.doi.org/10.6018/ analesps

Paba C. y González R. (2014). La actividad metacognitiva y la comprensión lectora en estudiantes de décimo grado. Psicologia desde el Caribe, 31(1), 79-102. Recuperado de http://rcientificas.uninorte.edu.co/index.php/psicolo $\mathrm{gia} /$ article/view/5185

Pérez, V. y La Cruz, A. (2014). Estrategias de enseñanza y aprendizaje de la lectura y escritura en educación primaria. Zona Próxima, (21). 1-16. doi: http://dx.doi.org/10.14482/psdc.31.1.5185

Perkins, D. (1995). Escuela inteligente. Barcelona: Gedisa.

Reigeluth, C. M. (2013). Instructional-design theories and models: A new paradigm of instructional theory, Volume II. New York, USA: Routledge. doi: https://doi.org/10.4324/9781410603784

Ruiz, J. (1999). Metodología de la Investigación Cualitativa. Bilbao, España: Universidad De Deusto.

Sandín, M. (2003). Investigación cualitativa en educación. Fundamentos y Tradiciones. España: Mc Graw and Hill Interamericana.

Secretaría de Educación Pública [SEP]. (2011). Plan de estudios 2011. Educación Básica. México: Secretaría de Educación Pública.

Silas C. J. y Gómez L. (2013). El desarrollo de habilidades lectoras en la escuela telesecundaria. Algunas reflexiones sobre el papel del docente y los logros de los alumnos. Revista de Investigación Educativa, 17, 66-87 Recuperado de http://hdl.handle.net/11117/806

Stake, R. (1998). Investigación con estudio de casos. España: Morata.

Tavakoli, H. (2014). The effectiveness of metacognitive strategy awareness in reading comprehension: The case of Iranian university EFL students. The Reading Matrix, 14(2), 314-336. Recuperado de http://www.readingmat rix.com/files/11-2405q41u.pdf

Vallés, A. (2005). Comprensión lectora y procesos psicológicos. Liberabit, 11, 49-61. Recuperado de http://pepsic.b vsalud.org/pdf/liberabit/v11n11/v11n11a07.pdf

Viana, F., Ribeiro, I. y Santos, S. (2014). Los desafíos de enseñar a comprender. Investigaciones sobre lectura, (1), 9-32. doi: https://doi.org/10.37132/isl.v0i1.9

Zermeño, M. G. G., Guzmán, S. M. y Arroyo, J. A. R. (2014). Investigación Diagnóstica "Curso Hibrido: Investigación Científica y Tecnológica”. Revista de Investigación Educativa del Tecnológico de Monterrey, 5(9), 66-79. Recuperado de http://rieege.tecvirtual.mx/

\section{BY-NC-ND}

